\title{
Sobre la existencia de los mundos posibles
}

\section{Omar Hildebrando Vásquez Dávila}

\author{
BA-Logic, Lógica y Filosofía de la Lógica
}

\begin{abstract}
Resumen
En la discusión acerca del estatus ontológico de los mundos posibles, las partes en disputa, a saber, el realismo modal y el actualismo modal, desacuerdan respecto del alcance de la noción de "existencia". Para los primeros el reino de lo existente abarca tanto los mundos posibles como los mundos actuales. ${ }^{1}$ Para los segundos, sólo el mundo actual existe efectivamente. Analizamos aquí la propuesta de Di Leo (2016), según la cual, la diferencia de valuación entre enunciados que contengan las nociones de "existencia actual" y "existencia" simpliciter rehabilitaría el realismo modal de Lewis (1986), el cual ha sido cuestionado por Barrio (1999).
\end{abstract}

\begin{abstract}
In the discussion about the ontological status of possible worlds, there are typically two different positions: modal realism and modal actualism. They disagree on the scope of the notion of 'existence'. According to modal realism, the kingdom of existence includes possible and actual worlds, while according to actualism, only the actual world really exists. We examine in this work Di Leo's (2016) proposal, according to which, there are different valuations between statements that include the notion of 'actual existence' and the statements that include the notion of 'existence' simpliciter. If it was true, it would represent a defense of Lewis' (1986) modal realism, which has been questioned by Barrio (1999).
\end{abstract}

\section{Mundos Posibles}

No es difícil imaginar que las cosas pueden ser, o podrían haber sido, de distinto modo. Los mundos posibles son, en principio, todos esos modos distintos en que las cosas pueden ser, o podrían haber sido. Estos modos o mundos distintos pueden entenderse de manera realista (modal realism) o de modo "actualista" (modal actualism). Según la primera postura, los mundos posibles existen de la misma manera en que existe el mundo real (o "actual"), están compuestos de objetos físicos como los de este mundo, pero en un espacio-tiempo distinto. Según los actualistas, los mundos posibles son entidades distintas al mundo real, son entidades de tipo abstracto, como los conjuntos.

\section{Palabras clave}

realismo modal actualismo necesidad posibilidad modalidad

1. Entiéndase actual, actualismo, actualmente como real, realismo, realmente, ie., despojado de su connotación temporal propia del significado de actual en español.

\section{Keywords}

modal realism actualism necessity possibility modality 
2. Por 'existencia efectiva' se entiende existencia real (actua). O sea, el tipo de existencia que tienen las cosas de este mundo. Realidad y existencia efectiva son términos co-extensivos para ambas partes en disputa, sin embargo, los actualistas consideran que hay cosas que no son reales, e.g., los mundos posibles.

3. Según Williamsom (2013), la polémica entre el actualismo y el posibilismo es oscura. Este autor propone reemplazarla por la disputa entre necesitismo y contingentismo. Véase más adelante la sección 3.2.

4. Es importante advertir que en la mayor parte de la literatura acerca de

la polémica actualismo-posibilismo

se reconstruye la posición actualista desde el punto de vista realista, lo cual es inevitablemente tendencioso y representa una desventaja para el actualismo.

5. Es cierto que Lewis niega que haya tal diferencia, pero esta idea de la dependencia tampoco explica en qué sentido esta diferencia es mera apariencia.
Ambas posturas suscribirían la existencia efectiva (genuina o real) ${ }^{2}$ y primordial de las cosas respecto de las maneras en las que podemos referirnos a ellas, i.e., el dominio lingüístico depende del dominio ontológico. Tal vez por esto es importante zanjar la cuestión de qué son los mundos posibles, ¿tienen existencia efectiva (real) o no?

Ahora bien, asociadas a estas descripciones básicas de ambas posturas, ${ }^{3}$ tenemos la tesis de la pluralidad de mundos y la tesis indexical de la actualidad, propuestas por David Lewis. Según la primera, cualquier modo posible en que un mundo podría ser, es un modo en que algún mundo es. El mundo actual, este mundo, sólo sería uno de los infinitos mundos posibles existentes. La segunda tesis permite un análisis relativista del término 'actual'. El adjetivo 'actual' es indexical, lo cual quiere decir que su referencia depende de la circunstancia de emisión. El mundo actual es el mundo en el que ocurre un acto de emisión lingüística. Así que cualquier mundo posible podría ser un mundo actual.

De esta segunda tesis se desprenden algunas intuiciones que dan cuenta de la noción de mundos posibles. ${ }^{4}$ En primer lugar, la indexicalidad permite individualizar el mundo en que vivimos, o, en su defecto, el mundo en el que un hablante hace una emisión lingüística. Tenemos así que todo mundo es actual en sí mismo (para sus hablantes), pero no todos los mundos son actuales. Un mundo es actual sólo para los que lo habitan. En segundo lugar, esta tesis permitiría explicar la dependencia de los mundos posibles respecto del mundo actual (cualquiera que éste sea). Los mundos posibles necesitan de un mundo respecto del cual sean posibles.

Esta segunda idea es independiente del actualismo o del realismo modal, ya que, en contra del actualismo, el mundo que permite la posibilidad de los llamados mundos posibles, no es necesariamente el mundo actual (o este mundo tal como lo conocemos), pues como se dijo, el mundo actual es relativo al que lo habita. Fijado un mundo, podemos hablar de mundos posibles y discutir si todos tienen el mismo estatus ontológico o no.

Por otro lado, esta idea tampoco juega mucho a favor del realismo modal, ya que no da cuenta de la intuición general, según la cual, hay una diferencia entre este mundo actual y todos los mundos posibles que dependen de él (o entre este mundo actual y los restantes mundos actuales posibles donde algún habitante emite una oración). ${ }^{5}$ Además, Eduardo Barrio ha sostenido que el realismo de Lewis no da cuenta de la diferencia entre "existir" y "existir actualmente" (Barrio, 1999: 10-15). Es legítimo preguntar por esta diferencia incluso si concediéramos que tanto los mundos actuales como los posibles tienen existencia efectiva (o real, genuina). Si fuese cierto que en la propuesta realista se asumiese que el dominio ontológico es primordial al dominio lingüístico, entonces la diferencia entre los mundos actuales y los mundos posibles tiene que ser real (metafísica) y no meramente lingüística como propone Lewis con su tesis de la indexicalidad.

A pesar de esto, se suele sostener que el análisis relativista del término 'actual' es un argumento a favor del realismo modal. Di Leo (2016) ha argumentado que enunciados como:

EA: Necesariamente, yo existo actualmente.

$E B$ : Necesariamente, yo existo.

servirían como ejemplo para mostrar que desde el realismo modal se puede satisfacer aquella exigencia intuitiva que ve una diferencia entre "existir" y "existir actualmente". Según Di Leo, estos enunciados tienen formas lógicas distintas, y el valor de verdad de $E A$ depende del alcance del operador de actualidad. En particular, $E A$ 
siempre es verdadero, en cambio, $E B$ puede ser falso. Sólo si aceptáramos esta diferencia, deberíamos aceptar estas dos cosas: a) la referencia al mundo actual presente en $E A$ es lo que determina su verdad en toda circunstancia de evaluación, y b) existe un mundo posible donde $E B$ no es verdadera, un mundo posible que no es el actual. Mostraré que $E A$ no es verdadero debido al operador de actualidad y que $E B$, suponiendo el sistema modal S5, siempre es verdadero. ${ }^{6}$

\section{La crítica al actualismo}

Según Lewis, los actualistas confunden existir con existir actualmente. Por eso, sostienen que todo lo que existe es actual. La única forma de refutar la tesis de la pluralidad de mundos, según Lewis, es mostrar que lo actual es todo lo que existe. Las posibilidades quedarían fuera de aquello que se considera que existe. Pero esto es lo que se debería justificar. Como esto no ha sido justificado, podríamos pensar que las posibilidades existen, si esto es así, lo posible sería parte de lo actual, y esto último, según Lewis, es inadmisible. Por otro lado, tampoco en el actualismo se acepta esto, ya que desde esta postura no se ve a las posibilidades como parte de la realidad, sino como alternativas de ella. La solución propuesta por los realistas consiste en decir que la existencia (real o genuina) está compuesta de las cosas que existen actualmente y de las cosas que existen posiblemente. Y describen la posición actualista como aquella que sólo otorga existencia real a las cosas que existen actualmente. ${ }^{7}$ Esta descripción convierte al actualismo en una postura trivialmente verdadera.

Lewis, tal vez para no ser catalogado como un multiplicador de entidades, ha destacado la simpleza y la economía de su propuesta. Acusa al actualista de tener distintas nociones de existencia: efectiva y no-efectiva (o no genuina). En cambio, en su propuesta "existe" se dice de una sola manera: existencia efectiva (o real). ${ }^{8}$ No es difícil ver, sin embargo, que la complejidad y el despilfarro ontológico simplemente se han desplazado al nivel de los mundos. Pero concediendo todos estos puntos, podemos todavía descartar la diferencia entre $E A$ y $E B$ propuesta por Di Leo y con la cual pretende defender la postura realista de Lewis.

\section{3. ¿Cómo interpretar EA y EB?}

\subsection{Lecturas de EA y EB}

$E A$ y $E B$ contienen un operador de modalidad que expresa necesidad, una expresión indexical ('yo') y el predicado de existencia. EA además posee la expresión actualmente (o sea, realmente). La diferencia entre estos enunciados está dada, entonces, por la aparición de esta noción en el primer caso, en $E A$. Si ignoráramos la aparición del indexical 'yo' (o de la referencia a la primera persona del singular), se evaluaría el valor de $E A$ mirando al mundo donde se hace la emisión. Por ejemplo, para determinar la verdad de "Necesariamente, María existe actualmente" debemos mirar este mundo, el mundo actual (real) y confirmar si María lo habita. Sin embargo, la aparición del indexical 'yo' hace que $E A$ sea una verdad casi de tipo analítica. No se necesita ni siquiera mirar al propio mundo, ya que si tenemos el enunciado "yo existo actualmente" es porque fue emitido por alguien, a saber, por mí. Este enunciado puede parafrasearse como:

$E A^{*}$ : Necesariamente yo existo donde hago esta emisión.

Ya que el mundo actual para los realistas es meramente eso, i.e., el lugar donde se hace la emisión. Siempre que tengamos a mano la oración "yo existo", va a ser verdadera. ${ }^{9}$
6. Debería aceptarse, de acuerdo a los principios del realismo modal, que si $E B$ siempre es verdadera, EA también lo es. Ya que el mundo actual al que $E A$ hace referencia es simplemente el mundo donde se hizo la emisión, y no guarda ninguna diferencia de tipo metafísico con el resto de mundos posibles.

7. Nos hemos deslizado aquí desde los mundos actuales y los mundos posibles, a las cosas u objetos que existen de manera actual o posible. Para Lewis los objetos posibles (no actuales) son tan reales como los mundos posibles. Sin embargo, la existencia de los mundos posibles no garantiza automáticamente la existencia de los objetos posibles. Para que esto sea cierto hay que precisar el dominio del discurso respecto al cual se entiende el predicado de existencia. Para la definición de objeto posible véase Lewis (1986: 211-212).

8. En otros términos, el actualista se ve forzado a dividir la noción de existencia entre existencia actual y existencia no-actual. Cfr., Lewis (1986: 98-100) y Lewis (1973: 84-91).
9. Dicho de otro modo, no tengo que buscar mi persona a lo largo de los mundos. Si la oración "yo existo" aparece, es suficiente para que sea verdadera. 
10. Esto nos permitiría usar alternativamente y sin problemas los enunciados "Yo existo de manera posible" y "Posiblemente yo existo", ya que serían equivalentes.
De este modo, $E A$ no necesita comprometerse con mundos posibles ni con ningún tipo de ontología. Ni siquiera requiere un análisis del alcance de los operadores de modalidad o de actualidad.

Para analizar $E B$, parafraseemos $E A$ y $E B$ del siguiente modo:

EA: Es necesario que yo exista en este mundo (en el actual, o sea, en el real).

$E B$ : Es necesario que yo exista.

Para el realismo modal lo que existe comprende al mundo actual y a los mundos posibles. De modo que $E B$ puede estar atribuyendo una existencia tanto actual como posible, una existencia sólo actual, o una existencia sólo posible. La segunda opción queda descartada, puesto que de ser esa la interpretación, estaríamos buscando una diferencia entre dos enunciados idénticos: no habría diferencia entre $E A$ y $E B$. Así que sólo tenemos dos modos de interpretar $E B$.

$E B_{1}$ : Es necesario que yo exista en el mundo actual y en los mundos posibles.

$E B_{2}$ : Es necesario que yo exista en algún mundo posible.

Si asumimos que existir en algún mundo posible es existir de manera posible, podemos interpretar $E B_{2}$ (y por lo tanto $E B$ ) como:

\section{$E B^{*}$ : Es necesario que yo exista de manera posible.}

Ahora bien, hemos mostrado anteriormente que $E A$ es siempre verdadera; y dado que $E B_{1}$ es la conjunción de $E A$ y $E B_{2}$, nuestra atención debe dirigirse a precisar el valor de verdad de $E B_{2}$ (o, lo que es lo mismo, de $E B^{*}$ ). Antes de hacer esto, veamos algunos principios de la lógica modal que nos ayudan a entender, más precisamente, $E B^{*}$.

\subsection{Necesitismo y contingentismo}

Se asume aquí, por simplicidad, el sistema modal S5 (cfr. Priest, 2008: cap. 3), en el que lo que es posible es necesariamente posible, y lo que es necesario es necesariamente necesario. Así, si es posible que yo exista, es necesariamente posible que yo exista. De manera de que si lo que está en negrita en $E B^{*}$ es verdadero, $E B^{*}$ es verdadero. Es importante también asegurarnos de que si algo con determinadas características (e.g., yo) pudiera existir, entonces, existe algo que pudiera cumplir con esas características, y la conversa también debe cumplirse. ${ }^{10}$ Esto nos permitiría entender $E B^{*}$ como: es necesario que posiblemente yo exista. Y para esto tenemos dos principios de la lógica modal: la fórmula de Barcan $(\mathrm{BF})$ y su conversa $(\mathrm{CBF})$, los cuales explicamos a continuación.

Timothy Williamson (2013) ha señalado que la disputa entre actualistas y posibilistas es oscura y que debería ser reemplazada por la disputa entre necesitismo (necessitism) y contingentismo (contingentism).

» Necesitismo (necessitism): necesariamente todo es necesariamente algo.

(NNE) $\quad \square \forall x \square \exists y x=y$

» Contingentismo (contingentism): es la negación de (NNE), i.e., posiblemente algo es posiblemente nada. 
Independientemente de que Williamson tenga razón respecto de la oscuridad de la disputa actualismo-posibilismo, es cierto que el realista modal comparte los principios $\mathrm{BF}$ y $\mathrm{CBF}$ con el necesitismo.

De (NNE) podemos derivar dos principios de la Lógica Modal de Primer Orden sostenidos por el necesitista: la fórmula de Barcan (BF) y su conversa (CBF):

(BF) $\quad \diamond \exists x A \rightarrow \exists x \diamond A$

(Si pudiera haber habido algo que cumpliera una condición, hay algo que pudiera haber cumplido esa condición)

(CBF) $\exists x \diamond A \rightarrow \diamond \exists x A$

(Si hay algo que pudiera haber cumplido una condición, podría haber habido algo que cumpliera la condición)

Así, estamos habilitados a entender $E B^{*}$ como: es necesario que posiblemente yo exista. Y, por último, bastaría con que fuese verdadero que posiblemente yo exista, para obtener la verdad de $E B^{*}$. Si $E B^{*}$ es siempre verdadero, cualquiera sea la interpretación de $E B$, siempre va a ser un enunciado verdadero. De este modo, Di Leo no estaría habilitado a decir que $E B$ puede ser falso.

\section{3. "Posiblemente yo existo" siempre es verdadero}

Si $E B^{*}$ prescindiera de la referencia a la primera persona, e.g., "Es necesario que María exista", analizaríamos su valor de verdad inspeccionando los mundos, accesibles desde el mundo actual, habitados por María. Si todos son habitados por María, el enunciado será verdadero, tanto si interpretamos 'existir' como existir en el mundo actual como si lo interpretamos con respecto a los mundos posibles. Si hay al menos un mundo que no sea habitado por María, el enunciado será falso. Puede darse el caso que María no exista en ningún mundo (o que exista en todos).

Sin embargo, $E B^{*}$, al contener la referencia a la primera persona, esta persona debe existir por lo menos en el mundo actual (y donde sea que se enuncie). Y como todos los mundos están relacionados con todos, basta que en un mundo "yo existo" sea verdadera para que resulte verdadera la oración "Posiblemente, yo existo". Si esta oración es verdadera, entonces es necesariamente verdadera, i.e., "Es necesario que sea posible que yo exista" es siempre verdadera, al igual que $E A$. De modo que, $E A$ y $E B$ reciben siempre el mismo valor de verdad. Si bien es cierto que esto se debe a distintas razones, ninguna de ellas representa una falta a los principios del realismo modal defendido por Lewis y Di Leo.

\section{Conclusión}

Hemos visto que la defensa de Di Leo del realismo modal de Lewis no cumple su propósito ya que sigue ausente una explicación, que no sea meramente lingüística, de la diferencia entre existir actualmente y existir simpliciter. En particular, los enunciados $E A$ y $E B$ propuestos para ejemplificar la diferencia entre "existir" y "existir actualmente", no cumplen con su objetivo. 


\section{Bibliografía}

" Barrio, E. A. (1999), "Indexicalidad y realism modal", en Cuadernos de Filosofía, 45, pp. 49-68.

"Di Leo, J. (2016), "Actualidad, indexicalidad y estatus ontológico", Cuadernos de Filosofía 67-68, pp. 115-124.

»Lewis, D. (1973), Counterfactuals, Blackwell Publishers, Oxford, 2001.

» Lewis, D. (1986), On the plurality of worlds, Blackwell, Malden.

» Priest, G. (2008), An Introduction to non-Classical Logic: From if to is. Cambridge University Press, New York.

"Williamson, T. (2013), Modal Logic as Metaphysics. Oxford: Oxford University Press. 